\title{
On the occurrence of $\{113\}$ facets on CVD-grown diamond
}

\author{
G. Janssen, J.J. Schermer, W.J.P. van Enckevort ${ }^{1}$ and L.J. Giling \\ Experimental Solid State Physics III, Unicersity of Nijmegen, 6525 ED Nijmegen, Netherlands
}

Received 8 May 1992; manuscript received in final form 17 July 1992

\begin{abstract}
Relatively thick films of diamond were deposited homoepitaxially on cylindrical substrates from an acetylene-oxygen combustion flame. On these single crystals a number of stable $\mathbf{F}$ faces are present. For the first time stable facets other than $\{111\}$ and $\{100\}$ are observed, namely $\{113\}$ facets. These facets occur under conditions of low surface coverage, e.g. at high deposition temperatures. This confirms the prediction of a previously developed crystal growth model. A preliminary investigation of the relative stability of the $\{113\}$ faces as function of the deposition temperature is described.
\end{abstract}

\section{Introduction and theory}

The power of sphere-growth experiments for the investigation of fundamental crystal growth phenomena, and especially the relative stability of the crystal faces, is well known [1-3]. Gardeniers and co-workers [4] have performed very extensive experimental and theoretical studies on the chemical vapour deposition (CVD) of silicon on hemispherical substrates. They observed only flat faces with indices $\{h h k\}$. The reason for this is the very high stability of the $\langle 110\rangle$ periodic bond chains (PBCs [5]), which are the basic entities of these $\{h h k\}$ faces. Because of this, all surfaces in the $\langle 110\rangle$ zones have a potential $S$ character. Gardeniers et al. argued that those crystallographic orientations, which correspond to the local minima of the surface tension, will appear as flat faces on a convex substrate after deposition of an epitaxial layer. From a simple first order broken bond model for crystals with the diamond structure it follows that only the $\{111\}$ faces are stable. This is also predicted by the PBC theory [5]. However, the surface tension of (100)-like surfaces can be lowered by the com-

\footnotetext{
1 Present address: Solid State Chemistry, University of Nijmegen, 6525 ED Nijmegen, Netherlands.
}

bination of two dangling bonds to form a dimer. This results in a $(1 \times 2)$ reconstruction, which is well known to exist on the $\{100\}$ faces of silicon (see, e.g., ref. [6]). Gardeniers et al. furthermore argued that a similar dimer formation can occur for all faces in the $[01 \overline{1}]$ zone with angles $\varphi$ in the region $\varphi_{111}<\varphi \leq \varphi_{100}$ (and symmetry-related parts of the zone). Including this surface reconstruction in the model they calculated that also $\{100\}$ and $\{113\}$ faces will appear as flat faces [7].

A further reduction of the surface tension can be brought about by the adsorption of gas species onto the dangling bonds of the surface. The absolute change in the surface tension is determined by the surface coverage and the density of adsorption sites on the surface. This means that different orientations can have a different behaviour of the surface tension as function of the surface coverage. Therefore, a face which has an $\mathrm{F}$ character at zero adsorption can turn into a $\mathrm{K} / \mathrm{S}$ face at high surface coverages. From the model calculations it was shown that exactly this phenomenon occurs for the reconstructed $\{113\}$ faces of silicon. The stabilizing effect of the dimers of the exact $\{113\}$ faces is compensated by the higher absolute density of adsorbed species on the slightly misoriented surfaces [7]. Thus, above a certain critical coverage the $\{113\}$ faces will become rough. The surface coverage will increase 
upon lowering the temperature, from which it can be concluded that the $\{113\}$ faces will not grow planar below a certain critical temperature. This temperature is called the "chemical roughening" temperature, to distinguish it from the well-known "thermal roughening" temperature (see, e.g., ref. [8]). Similarly, the surface coverage and thus the appearance of the $\{113\}$ faces can be changed by varying the composition of the gas phase, in the case of silicon deposition, by changing the $\mathrm{Cl} / \mathrm{H}$ ratio [9].

The observed, relative stability of the $\{113\}$ faces as a function of the temperature and the $\mathrm{Cl} / \mathrm{H}$ ratio [1,9] is in remarkably good agreement with these theoretical predictions. For total surface coverages $\left(\theta_{\text {total }}\right)$ below approximately 0.8 these facets are flat $F$ faces. That is, the (polar) plot of the surface tension versus the crystallographic orientation shows a cusped minimum in all directions for the $\{113\}$ faces. For $\theta_{\text {total }} \geq 0.8$ only macrosteps parallel to the $\langle 1 \overline{1} 0\rangle$ PBCs are observed, while the surface is curved in the perpendicular direction. This implies that the surface tension has a cusped minimum only in the direction parallel to the PBC. Or in other words: at low surface coverage all steps on a $\{113\}$ face have positive step free energies and thus will not form, while for high surface coverages the step free energy becomes negative for all steps except those parallel to the $\langle 1 \overline{1} 0\rangle \mathrm{PBC}$. In this last case an $S$ face has been formed. Although not explicitly stated in the theoretical studies of Gardeniers on silicon [4], it can be derived from this work that under most growth conditions all faces in the $\langle 1 \overline{1} 0\rangle$ zones are $S$ faces with steps parallel to the in-plane PBC. Therefore, these regions will appear as curved, stepped $\mathrm{S}$ zones in virtually all sphere growth experiments. This is quite understandable, because in order to form steps perpendicularly to the $\mathrm{PBC}$ and thus give the face a $\mathrm{K}$ character, the stable PBCs should be broken up which requires large amounts of energy.

A few more remarks are in place here. Firstly, the theory on the stability of different faces was derived under the assumption of near-equilibrium crystal growth, that is for low supersaturations. Secondly, it should be noted that after a sufficiently large crystal has developed only facets corresponding to the absolute minima in the surface tension will remain. All other faces, which we have called flat and stable here, are in fact only present in a transition phase from the original seed to the final crystal. Thirdly, unfortunately the theory does not predict the occurrence of $\{110\}$ facets and the wealth of facets with higher Miller indices (e.g. \{337\}, \{331\} and $\{551\}$ ) that can be observed experimentally on hemispheres overgrown by silicon CVD [1].

As diamond and silicon have the same crystal structure and the $(1 \times 2)$ reconstruction is known to occur for diamond as well $[6,10,11\}$, the above summarized theory could also apply to diamond. Without going through the surface tension calculations again it is clear that the same general rules should govern the growth of diamond at least in a qualitative manner. Indeed planar $\{111\}$ and $\{100\}$ faces are generally observed on polycrystalline diamond films. To the best of our knowledge no other $F$ faces were ever reported for CVD grown diamond. This is not very surprising as it is rather difficult to determine the crystallographic orientation of the individual faces in a polycrystalline layer. The crystals in such layers are often truncated and the shape of the facets can be influenced by interactions with the neighbouring crystals and by twinning. Moreover, these tiny crystals have already reached their final shape, with only those faces present that correspond to the absolute minima in the growth rate.

Based on the theoretical calculations described above the $\{113\}$ facets are expected to develop under growth conditions where the crystal surfaces are relatively clean. However, during normal diamond deposition the conditions are such that the surface will be almost completely covered by hydrogen and the growth precursors [1214]. This is caused by the extreme non-equilibrium condition of the gas phase necessary for good-quality diamond deposition. The gas phase concentrations of both atomic hydrogen and many hydrocarbon radicals are often orders of magnitude higher than the heterogeneous equilibrium concentrations at the deposition temperature. All of these radicals have high sticking probabilities on the diamond surface. The desorption rate of the radicals is low because of the relative low 
deposition temperatures $\left(800-1000^{\circ} \mathrm{C}\right)$. These reasons lead to a surface coverage very close to unity, which would inhibit the occurrence of $\{113\}$ faces. Indeed we have reported on a spheregrowth experiment in a hot filament assisted CVD system, for which no $\{113\}$ facets were observed [3]. The surface coverage during this experiment at a deposition temperature of $920^{\circ} \mathrm{C}$ was certainly above 0.8 [14].

Recently it was shown that good quality, homoepitaxial deposition of diamond can also be performed at much higher temperatures (1200$1500^{\circ} \mathrm{C}$ ) in high power, high growth rate environments such as an acetylene-oxygen flame [15] or a plasma torch [16]. With this we arrive at the objective of the present work: can $\{113\}$ faces be formed in diamond sphere-growth experiments under conditions of low surface coverage?

\section{Experimental setup}

An acetylene-oxygen flame deposition setup was employed, which has been under development in our group for the last two years [17]. In this system, the deposition temperature can be controlled (within $\pm 10^{\circ} \mathrm{C}$ relative) and measured (within $\pm 20^{\circ} \mathrm{C}$ absolute) with considerable accuracy during long term experiments. Because of the large temperature and concentration gradi-
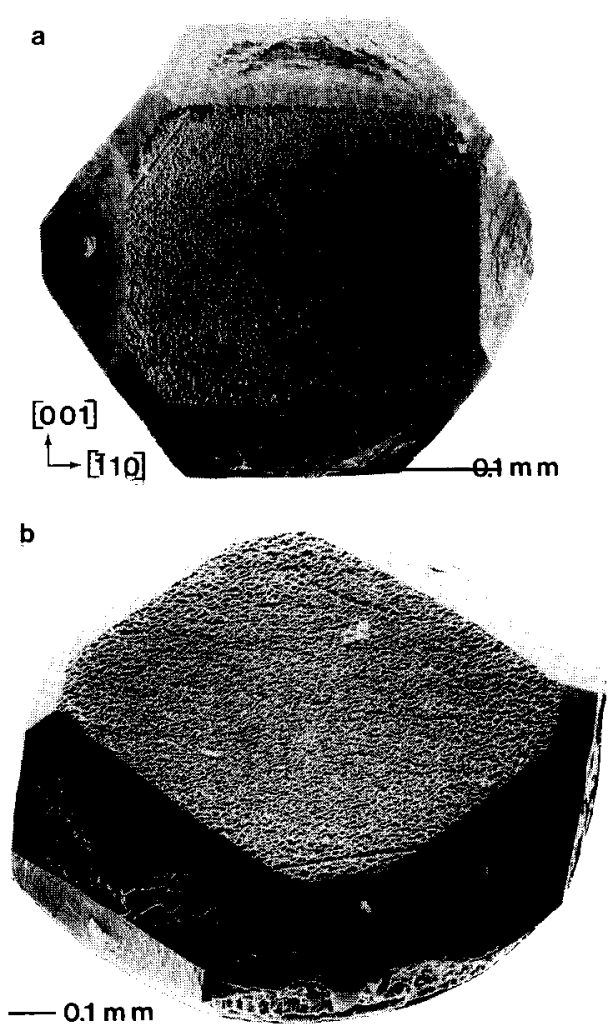
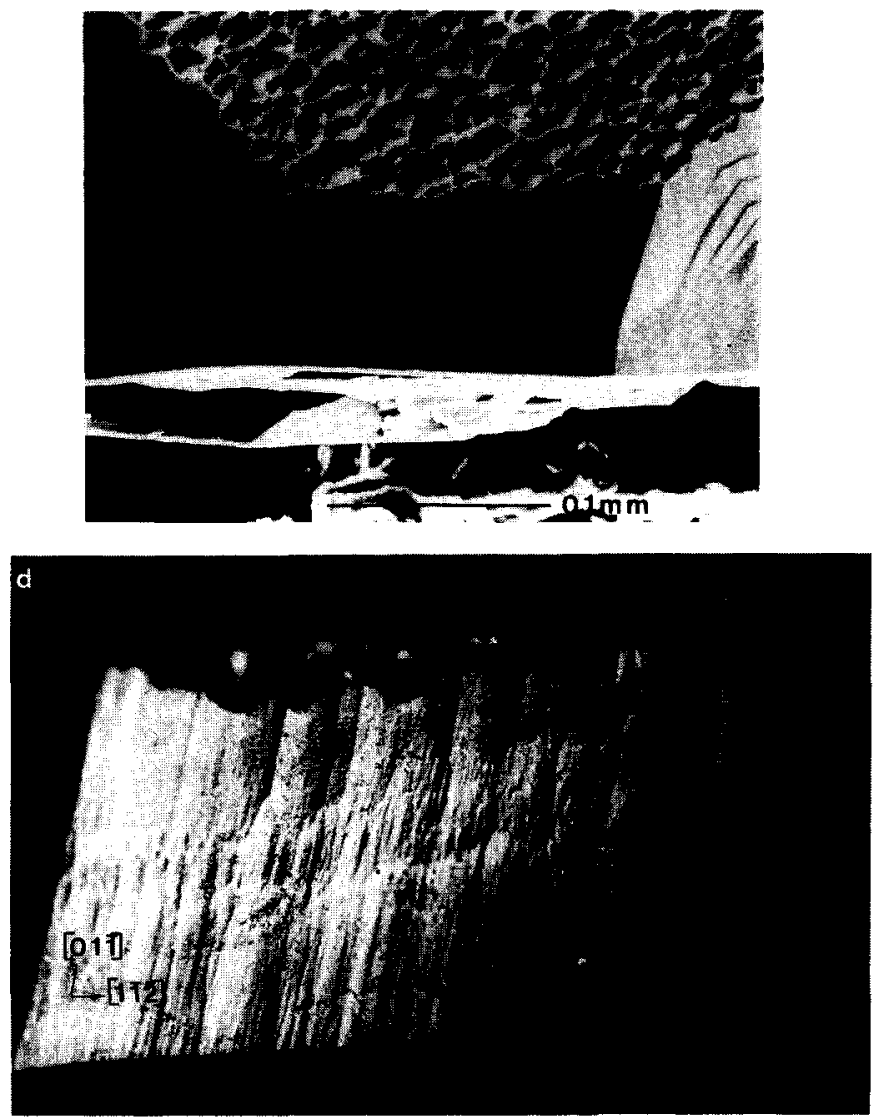

Fig. 1. Micrographs of a single crystal diamond grown in $1 \mathrm{~h}$ at $1190^{\circ} \mathrm{C}$ and $S_{\mathrm{c}}=2.9 \%$. (a) SEM micrograph viewing along [ $\left.\overline{1} \overline{1} 0\right]$ direction. (b) SEM micrograph at an angle of $\approx 30^{\circ}$ with the [110] direction, that is roughly in top view of the (311) facet. (c) SEM micrograph of the (311) facet, in top view. (d) Nomarski differential interference contrast micrograph of the same (311) facet as in 
ents existing in the flame, a variant of the sphere growth experiment was used, namely a "cylinder growth" experiment [18]. A thin cylinder $(0.25$ $\mathrm{mm}$ thick) was laser-cut from a slice of natural type IIa diamond. The base of the cylinder was chosen to have an (110) orientation (less than $2^{\circ}$ off). Therefore, the wall of the cylinder exhibits all possible orientations in the [110] zone, while on the rim of the cylinder all orientations between this zone and the (110) plane are available. The wall of the cylinder is not smoothly curved but somewhat corrugated. This is caused by the laser cutting process which produces grooves, approximately $20 \mu \mathrm{m}$ wide and $4 \mu \mathrm{m}$ deep, perpendicular to the base plane. Because of the high growth rates (typically $150 \mu \mathrm{m} / \mathrm{h}$ for the (110) faces) and the relative thick layers deposited (typically $\geq 60 \mu \mathrm{m}$ for all orientations), the roughness of the cylinder wall does not hamper the experiments. The base of the cylinder had a surface roughness of less than $20 \mathrm{~nm}$. The cylinder was soldered to a molybdenum substrate holder ensuring a good thermal contact. The diamond was placed in the central axis of the flame. For polycrystalline diamond films deposited under similar conditions in one hour or more, it was found that the central area (diameter $3 \mathrm{~mm}$ ) of these films is homogeneous within $10 \%$, both in quantity and in quality [17]. By using small cylinders, which had a diameter of only $1 \mathrm{~mm}$, uniform growth conditions over the complete cylinder could be ensured. Indeed, in none of the experiments were peculiarities caused by any inhomogeneity in the growth observed for the crystal surfaces directly facing the flame.

\section{Results and discussion}

On a number of these cylinders, a homoepitaxial film with a thickness between 60 and $150 \mu \mathrm{m}$ was deposited. The range of the deposition conditions is given in table 1. The deposits were investigated by SEM and optical - Nomarski differential interference contrast - microscopy. Figs. 1a and 1b show, in top-view and at an angle of $\approx 30^{\circ}$, respectively, SEM micrographs of a diamond crystal grown in $1 \mathrm{~h}$ at $1190^{\circ} \mathrm{C}$, using a
Table 1

Details of experimental setup and the range of deposition conditions used

\begin{tabular}{ll}
\hline Welding torch & $\begin{array}{c}\text { No. } 1 \text {; with a } 1 \mathrm{~mm} \\
\text { diameter orifice } \\
1.4-1.8 \text { Standard } \\
\mathrm{O}_{2} \text { flow }\end{array}$ \\
$\begin{array}{l}\text { liter per minute } \\
\text { Carbon supersaturation }\left(S_{\mathrm{c}}\right)\end{array}$ & $1.9-2.9 \%$ \\
Deposition temperature & $1120-1320^{\circ} \mathrm{C}$ \\
Deposition time & $0.5-4 \mathrm{~h}$ \\
Distance substrate to inner & $1.2-1.4 \mathrm{~mm}$ \\
$\quad$ flame & Natural type IIa diamond; \\
Substrate & $\{110\} ;$ Cylinder diameter \\
& $1 \mathrm{~mm}, 0.25 \mathrm{~mm}$ thick \\
\hline
\end{tabular}

carbon supersaturation $S_{\mathrm{c}}$ [19] of $2.9 \%$ (sample 1). The thickness of the layer in the [110] direction is $150 \pm 10 \mu \mathrm{m}$. In the top-view, the crystal has a truncated octagonal shape, as was also observed by Snail and Hanssen [15]. The (110) base face is still present and has a cobbled structure, as is typical for this $\mathrm{K} / \mathrm{S}$ face (refs. $[16,19,20]$ and references therein). Actually, for all samples investigated here, the (110) face consists of small, homogeneously distributed pits, typically 5 to 10 $\mu \mathrm{m}$ across, with planar side walls. Most probably these side walls have $\{100\}$ and/or $\{111\}$ orientations, but a more elaborate study is necessary to determine the exact orientations.

Along the rim of the cylinder a number of flat facets have developed (fig. 1). The boundaries of all these facets are sharp. An exception are all the boundaries with the original (110) face, which reflect the pitted structure of the (110) face. Also the boundaries of faces with the bottom part of the seed crystal are not well defined, probably because of problems in the transport of growth species towards the lower parts of the seed crystal. The crystallographic orientations of the facets can be determined by X-ray analysis or optical goniometry angle measurements, but these methods are rather cumbersome. The orientations can be determined much faster from the crystal and surface morphology in relation to the crystallographic symmetry, as will be shown below. The orientations of the main facets of the sample in fig. 1 were also verified by $\mathrm{X}$-ray analysis. For 


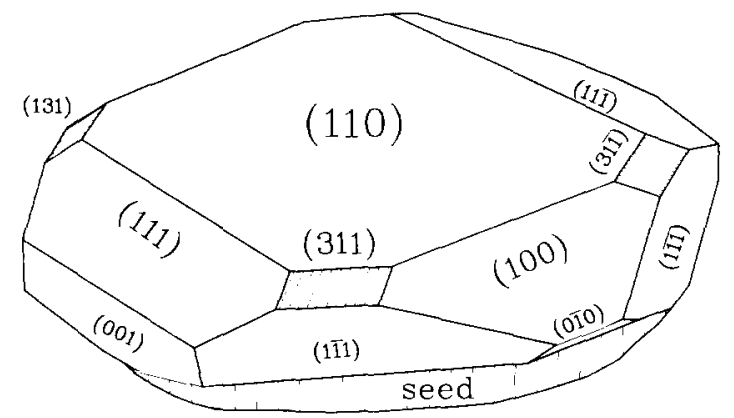

Fig. 2. Schematic overview of the diamond crystal shown in fig. 1 .

clarity a schematic overview of all the observed faces is given in fig. 2 .

Firstly, two sets of, respectively, two and four \{111\} $F$ faces can be easily identified by the occurrence of trigonal growth hillocks and the beginning of crack formation $[3,21]$. The growth hillocks are bounded by steps in the $|11 \overline{2}|$ directions (see ref. [3] for $|h k l|$ notation of in-plane, equivalent directions). The first set contains two facets, which make an angle of $35^{\circ}$ with respect to the (110) plane, while the four facets in the other set are perpendicular to the (110) plane. The facets of the last set are very rough with high terraces, rimmed by macrosteps and they also show some evidence of micro-twinning.

Secondly, three sets containing two $\{100\} \mathrm{F}$ faces each are present. They can be identified by the occurrence of square growth hillocks and square growth spirals with (macro)steps in the |011| directions. Two of these facets make an angle of $45^{\circ}$ with the (110) plane, two facets are perpendicular and two facets make an angle of $135^{\circ}$ with the (110) plane. Especially the presence of these last two facets is remarkable, as they are completely shielded from the main gas flow of the flame. All six $\{100\}$ facets are very similar in surface roughness and the appearance of the macrosteps.

Thirdly, four small facets with $\{113\}$ orientations are observed in between the large $\{111\}$ and $\{100\}$ faces. The orientation was deduced from careful measurements of the angles between these facets and the surrounding faces as well as from the directions of the boundaries between facets.
All four $\{113\}$ facets make an angle of $31.5^{\circ}$ with the (110) plane, and angles of $25.2^{\circ}$ and $29.5^{\circ}$ with the neighbouring $\{100\}$ and $\{111\}$ faces, respectively. Fig. 1c is a close-up of fig. 1b showing the (311) facet in more detail. The rims of this facet are parallel to $[01 \overline{1}]$ and $[1 \overline{1} \overline{2}]$ directions. The angle between the rims determined for all four facets was $75^{\circ} \pm 3^{\circ}$, in good agreement with the theoretical value of $73.2^{\circ}$. Inspection by differential contrast microscopy of the same facet (fig. 1d) shows that the facet is covered by macrosteps. Such macrosteps, parallel to the in-plane $\langle 1 \overline{1} 0\rangle$ PBC, were observed on all $\{113\}$ facets. Most of the higher macrosteps run across the full width of the facet, but some lower steps seem to be confined to the central region of the facet. From focusing the optical reflection microscope it was determined that the facets are slightly curved perpendicular to the $\mathrm{PBC}$ direction. The angle enclosed by the ( $180 \mu \mathrm{m}$ wide) facet is $2.5^{\circ}$, which is considerably smaller than the $20.7^{\circ}$ for the original seed crystal. Still, the presence of this curvature shows that the stabilizing effect perpendicularly to the [011] $\mathrm{PBC}$ is relatively weak.

No $\{110\}$ facets, apart from the starting (110) face, nor any other flat face - not mentioned above - were observed on this specimen and most other specimens. The growth rate in the $\langle 110\rangle$ directions $\left(R_{110}\right)$ for this crystal was $150 \pm$ $10 \mu \mathrm{m} / \mathrm{h}$. From the sizes of the different $\mathrm{F}$ faces, the relative growth rate of a $\{h k l\}$ face $\left(X_{h k l}=\right.$ $\left.R_{h k l} / R_{110}\right)$ can be determined. In this case $X_{111}$ $=0.39 \pm 0.08, X_{100}=0.46 \pm 0.08$ and $X_{113}=0.6$ \pm 0.1 .

The results described above were reproduced for a number of samples. The deposition conditions were varied slightly in these experiments. Although the size of the facets and thus their relative stability changed slightly between different samples, we were not able to find a clear relation with any of the growth parameters from this preliminary investigation. This probably means that most parameters are in someway coupled to each other. In addition, it was not possible to draw conclusions from experiments in which the growth conditions were changed more dramatically. Such experiments resulted either in rather thin deposits with undeveloped facets, or 
in a completely deteriorated growth with badly shaped or non-distinguishable facets. The only exception were the experiments in which the deposition temperature was varied.

As the transformation of the $\{113\}$ face is governed by the surface coverage, the stability of this face should change with the deposition temperature. For this reason, a series of experiments with different deposition temperatures in the range 1120 to $1320^{\circ} \mathrm{C}$ at an $S_{\mathrm{c}}$ of $1.9 \%$ and a $1 \mathrm{~h}$ deposition time were performed. For deposition temperatures above $\approx 1225^{\circ} \mathrm{C}$, the samples have the same characteristics as described above, with nicely formed $\{113\}$ facets. For temperatures below $\approx 1225^{\circ} \mathrm{C}$, no $\{113\}$ facets can be observed, but a curved band has appeared on the rim of the cylinder between the $\{100\}$ and $\{111\}$ facets. This band consists of high macrosteps parallel to the [011] PBC. In the direction of the PBC the band is flat, from which it is deduced that all $(h k k)_{h \geq k}$ orientations in the [011] zone, between and including (100) and (111), are present here. The occurrence of this $\mathrm{S}$ zone strongly confirms the stabilizing effect of the $\langle 1 \overline{1} 0\rangle$ PBCs [7], as mentioned in the foregoing description of silicon. The crystal deposited at $1225 \pm 20^{\circ} \mathrm{C}$ has an interme-
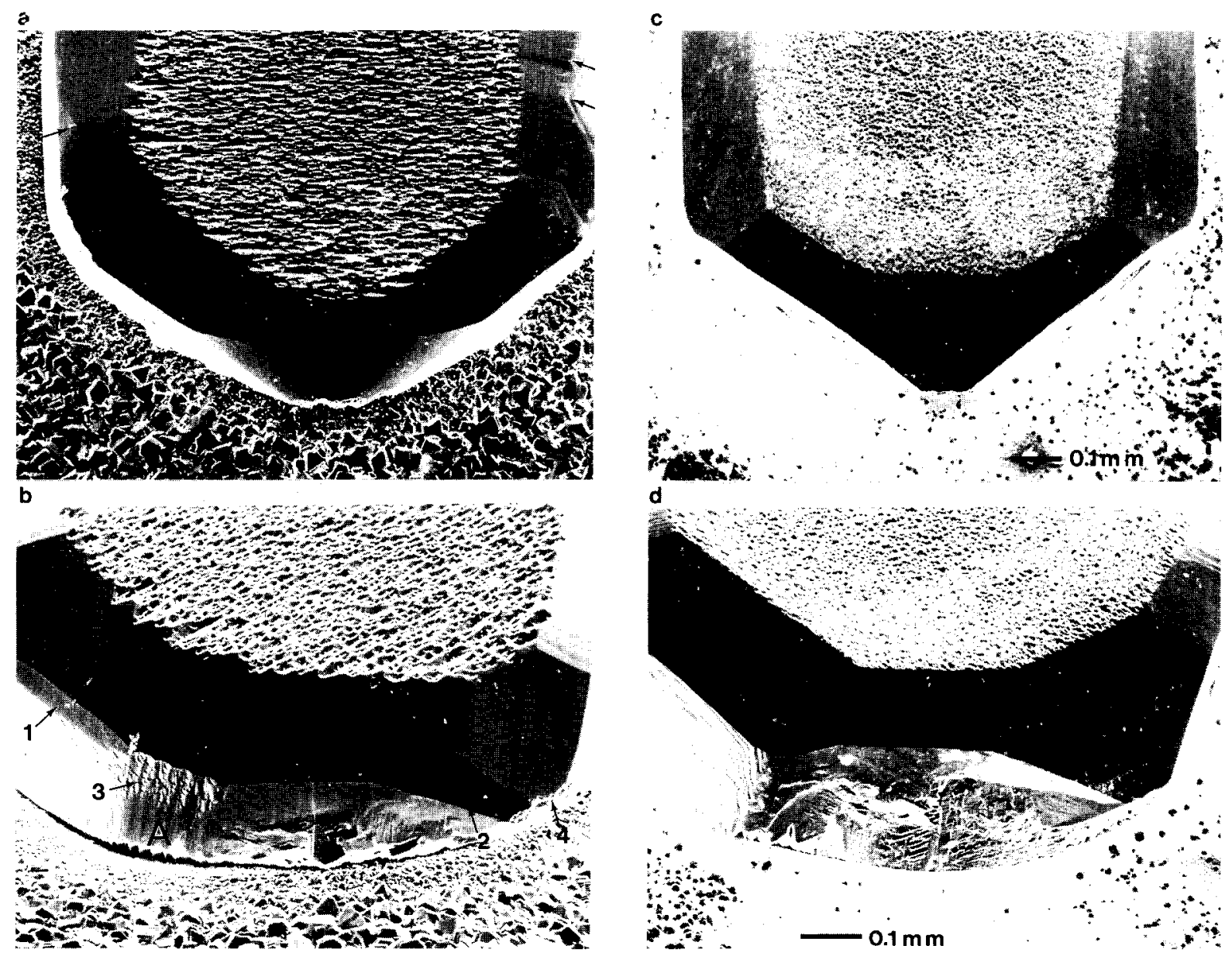

Fig. 3. SEM micrographs of single crystal diamonds deposited in $2.5 \mathrm{~h}$ at $1120^{\circ} \mathrm{C}$ (a, b), and in $1 \mathrm{~h}$ at $1320^{\circ} \mathrm{C}$ (c, d), both with $S_{\mathrm{c}}=1.9 \%$. Pictures (a) and (c) look down on the (100) face at an angle of $\approx 20^{\circ}$ with respect to the [110] direction. Pictures (b) and

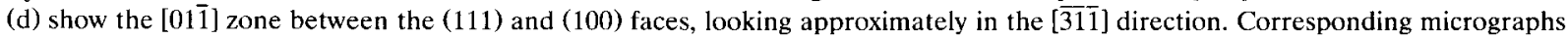
have the same magnification. 
diate structure, with small $\{113\}$ facets forming a part of a curved band.

The interpretation of these experiments in terms of the stability of the $\{113\}$ faces and the chemical roughening temperature is, however, complicated by the fact that the layers deposited on different samples do not have the same thickness. In the temperature range investigated, the growth rate in the $\langle 110\rangle$ directions increases with approximately $30 \mu \mathrm{m} / \mathrm{h}$ for every $100^{\circ} \mathrm{C}$ increase in the deposition temperature. The layers deposited on the rim of the cylinder are roughly 30 $\mu \mathrm{m}$ thick for the lowest deposition temperatures and $\approx 65 \mu \mathrm{m}$ thick for the highest temperatures. In determining these layer thicknesses it is assumed that the growth rate of all other faces behaves similarly as a function of the temperature as the growth rate of the $\{110\}$. The relatively thin layers, deposited at low temperatures, in combination with the small differences in the

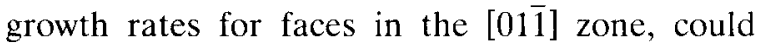
prevent the development of well-shaped $F$ faces and thus obscure the determination of the chemical roughening temperature. For this reason the two experiments at the extremes of the temperature range have been repeated in such a manner that the total layer thickness deposited on both samples was the same, by changing the deposition time.

The results for the low temperature sample $\left(T_{\text {dep }}=1120^{\circ} \mathrm{C}\right.$, time $\left.=2.5 \mathrm{~h}\right)$ and the high temperature sample $\left(T_{\text {dep }}=1320^{\circ} \mathrm{C}\right.$, time $\left.=1 \mathrm{~h}\right)$ are shown in fig. 3 . The layer thickness of the (110) face is $135 \pm 5 \mu \mathrm{m}$ for both samples. Although the layer deposited on the rim of the cylinder is much thicker now $(\approx 65 \mu \mathrm{m})$, still a curved $S$ zone is present on the low temperature sample. The angle enclosed by this band is quite large $\left(28^{\circ} \pm 4^{\circ}\right)$, compared to the previously observed value for the $\{113\}$ faces, and only a factor of two lower than the original curvature of the seed crystal (enclosed angle $48^{\circ} \pm 2^{\circ}$ ). The high temperature sample shows nicely shaped, flat $\{113\}$ facets. Obviously the complication of the determination of the chemical roughening temperature by the variation in layer thickness was not as dramatic as expected. However, another complication exists. Two new facets appear on the low temperature sample, which are indicated by the arrows ( 1 and 2$)$ in fig. $3 \mathrm{~b}$. It is rather difficult to identify the exact orientations of these facets, but from their position on the crystal it follows that facets 1 and 2 must be very close to the (113) and (311) faces, respectively. If this interpretation is correct, it would mean that the chemical roughening temperature is not the same for all $\{113\}$ faces on this crystal, which would be in conflict with the $\mathrm{m} \overline{3} \mathrm{~m}$ point group of diamond. However, small variations in the deposition conditions for the faces located at different positions on the same crystal might account for this phenomenon. Another possible solution to this discrepancy are in the laser grooves of the seed crystal, which are roughly parallel (after tilting towards the [110]) to

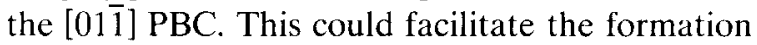
of high macrosteps consisting of small (100) and (111) faces for deposition conditions at which the surface tension of the (311) face and its surrounding faces is very similar to each other. These macrosteps could then obscure the (311) face. This reasoning is confirmed by the absence of a flat (113) face (indicated by $\mathrm{A}$ in fig. $3 \mathrm{~b}$ ) in the [110] zone. This area also shows high macrosteps. On the other hand, some unexplained discrepancy remains as no (113) or ( $\overline{3} 11)$ faces are present on the high temperature samples where the (311) face is much more stable. It should be mentioned that the transition from an $F$ to an $S$ face observed in silicon CVD was also rather gradually, with an intermediate morphology having hardly detectable $\{113\}$ faces [9].

Two more, interesting observations can be made about the crystal grown at low temperatures. Firstly, there exist regions around the (101) and $(1 \overline{1} 0)$ orientations, indicated by arrows ( 3 and 4 ) in fig. 3b, which have the same pitted structure as the (110) face. Probably the pitted structure stabilizes these faces. This gives some evidence to the idea that the wall of the pits have orientations corresponding to stable $F$ faces. In the (101) region it can be observed that the original grooves of the seed crystal still prevail in the final structure. Secondly, very large growth hillocks crossing the full width of the facets are present on the (111) and (11 $\overline{1})$ faces, as indicated by the arrows in fig. 3a. Remarkably the step directions of slow- 
est growth corresponding to these hillocks, $|\overline{1} 12|$, are inverted with respect to the steps normally present on $\{111\}$ diamond. This can be explained by the occurrence of step reconstruction [22].

From our experiments we deduce that the chemical roughening temperature for the $\{113\}$ faces of flame grown diamond lies around $1200 \pm$ $100^{\circ} \mathrm{C}$. The uncertainty is estimated from comparing the crystal in fig. 1 with the crystals in the temperature series and from the discrepancy in the formation of different $\{113\}$ faces on the same crystal. Of course, the temperature above which $\{113\}$ facets start to appear is not only dependent on the deposition temperature but also on the other growth conditions such as the input gas composition and the position of the seed crystal in the flame. A more elaborate study seems worthwhile, on the condition that better types of substrates - either hemispheres or cylinders with smooth walls - are used.

\section{Conclusion}

In conclusion we have shown for the first time that, apart from the well-known $\{111\}$ and $\{100\}$ faces, also $\{113\}$ facets can be formed on diamond by CVD techniques. These $\{113\}$ facets and their relative stability have originally been predicted by a crystal growth theory developed for silicon CVD. The described experiments show that the influence of surface reconstruction and surface coverage on the crystal growth described by this theory apply also to the deposition of diamond, at least in a qualitative way. A quantitative correlation between the experimental results and the theoretical model would require detailed knowledge either experimental or theoretical - about the composition of the gas phase at the growing crystal, in order to find the surface coverage of the diamond. The observation that a theory derived under the assumption of near-equilibrium growth conditions applies so well to our experiments is somewhat surprising. Two conflicting conclusions are possible: (1) The theory could be valid for a much wider range of conditions, even for the CVD of diamond which is generally assumed to be a far from equilibrium process. (2) Much more fascinating would be the conclusion that diamond deposition is a near-equilibrium process at the growth conditions used in this study. In a forthcoming paper a more elaborate theoretical and experimental comparison between diamond and silicon deposition will be made [14].

\section{Acknowledgements}

The authors wish to thank Mr. G.C.J. Otter and Ing. W. Vollenberg for their technical assistance. The diamond workers at Drukker International BV, Netherlands, are thanked for the preparation of the diamond seeds. This work was performed as part of the research program of the Netherlands Technology Foundation (STW) with financial support from the Netherlands Organization for Scientific Research (NWO).

\section{References}

[1] J.G.E. Gardeniers, W.E.J.R. Maas, R.Z.C. van Meerten and L.J. Giling, J. Crystal Growth 96 (1989) 821.

[2] J.G.E. Gardeniers, C.H. Klein Douwel and L.J. Giling, J. Crystal Growth 108 (1991) 319.

[3] W.J.P. van Enckevort, G. Janssen and L.J. Giling, J. Crystal Growth 113 (1991) 295.

[4] J.G.E. Gardeniers, Crystal Habit of CVD-Grown Silicon in Relation to Adsorption Processes, Thesis, University of Nijmegen (1990). Refs. $[1,2,7,9]$ are part of this work.

[5] P. Hartman, Z. Krist. 121 (1965) 78.

[6] L.J. Giling and W.J.P. van Enckevort, Surface Sci. 161 (1985) 567.

[7] J.G.E. Gardeniers, W.E.J.R. Maas, R.Z.C. van Meerten and L.J. Giling, J. Crystal Growth 96 (1989) 832.

[8] P. Bennema and J.P. van der Eerden, in: Morphology of Crystals, Ed. I. Sunagawa (Terrapub, Tokyo, 1987) p. 1.

[9] J.G.E. Gardeniers, M.M.W. Mooren, M.H.J.M. de Croon and L.J. Giling, J. Crystal Growth 102 (1990) 233.

[10] A.V. Hamza, G.D. Kubiak and R.H. Stulen, Surface Sci. $237(1991) 35$.

[11] T. Tsuno, T. Imay, Y. Nishibayashi, K. Hamada and N. Fujimori, Japan J. Appl. Phys. 30 (1991) 1063.

[12] G. Janssen, W.J.P. van Enckevort and L.J. Giling, in: Proc. Electrochemical Society 1st Intern. Symp. on Diamond and Diamond-Like Films, Los Angeles, 1989, Ed. J.D. Dismukes (Electrochemical Society, Princeton, NJ, 1989) p. 508.

[13] S.J. Harris, Appl. Phys. Letters 56 (1990) 2298.

[14] G. Janssen, J.G.E. Gardeniers and L.J. Giling, Diamond and Related Materials, in preparation. 
[15] K.A. Snail and L.M. Hanssen, J. Crystal Growth 112 (1991) 651.

[16] K.A. Snail, C.M. Marks, Z.P. Lu, J. Heberlein and E. Pfender, Mater. Letters 12 (1991) 301.

[17] J.J. Schermer, J.E.M. Hogenkamp, G.C.J. Otter, G. Janssen, W.J.P. van Enckevort and L.J. Giling, presented at Diamond 1992, Heidelberg, 1992.

[18] C.H.J. van den Brekel. J. Crystal Growth 23 (1974) 259.

[19] G. Janssen, W.J.P. van Enckevort, W. Vollenberg and L.J. Giling, Diamond and Related Materials 1 (1992) 789.
[20] G. Janssen, W.J.P. van Enckevort, J.J.D. Schaminée, W. Vollenberg, L.J. Giling and M. Seal, J. Crystal Growth 104 (1990) 752.

[21] W.J.P. van Enckevort, G. Janssen, W. Vollenberg, M. Chermin, L.J. Giling, J.J.D. Schaminée and M. Seal, Surface Coating Technol. 47 (1991) 39.

[22] W.J.P. van Enckevort and L.J. Giling, J. Crystal Growth $45(1978) 90$. 